\title{
Operation and Performance of the Mars Exploration Rover Imaging System on the Martian Surface
}

\author{
Justin N. Maki \\ Jet Propulsion Laboratory \\ California Institute of Technology \\ Pasadena, CA USA \\ Justin.N.Maki@jpl.nasa.gov \\ Todd Litwin, Mark Schwochert \\ Jet Propulsion Laboratory \\ California Institute of Technology \\ Pasadena, CA USA \\ Ken Herkenhoff \\ United States Geological Survey \\ Flagstaff, AZ USA
}

\begin{abstract}
The Imaging System on the Mars Exploration Rovers has successfully operated on the surface of Mars for over one Earth year. The acquisition of hundreds of panoramas and tens of thousands of stereo pairs has enabled the rovers to explore Mars at a level of detail unprecedented in the history of space exploration. In addition to providing scientific value, the images also play a key role in the daily tactical operation of the rovers. The mobile nature of the MER surface mission requires extensive use of the imaging system for traverse planning, rover localization, remote sensing instrument targeting, and robotic arm placement. Each of these activity types requires a different set of data compression rates, surface coverage, and image acquisition strategies. An overview of the surface imaging activities is provided, along with a summary of the image data acquired to date.
\end{abstract}

Keywords: Imaging system, cameras, rovers, Mars, operations.

\section{Introduction}

\subsection{Mission}

In January 2004, the Mars Exploration Rover Project (MER) landed a pair of Rovers onto the surface of Mars [1]. Each rover (see figure 1) carried a set of 10 cameras [2]. Upon landing, the cameras immediately began acquiring images of the Martian terrain and returned over 70,000 individual images in the first Earth year of operation. The cameras have made significant contributions to the mission, including the acquisition of images that show evidence of past liquid water on the Martian surface [3]. This paper

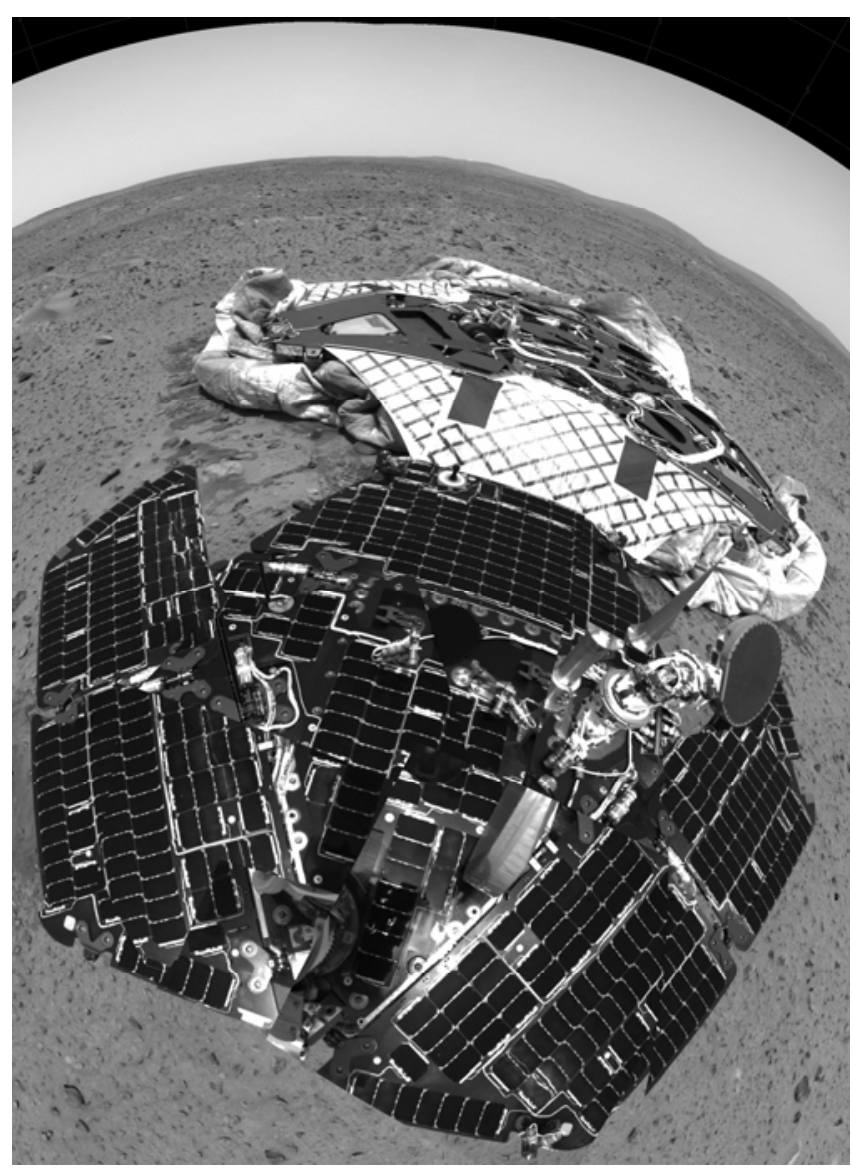

Figure 1. The Mars Exploration Spirit Rover, as viewed by the Navcam shortly after lander egress early in the mission.

presents an overview of the operation and performance of the MER Imaging System.

\subsection{Imaging System Design}

The MER cameras are classified into five types: Descent cameras, Navigation cameras (Navcam), Hazard Avoidance cameras (Hazcam), Panoramic cameras (Pancam), and Microscopic Imager (MI) cameras. The Descent camera, attached to the landing system, acquired 3 images during the Entry, Descent, and Landing (EDL) phase of the mission and was subsequently abandoned as the rover drove onto the surface. The other nine cameras are attached to the rover - the Navcam and Pancam cameras are mounted at the top of a pan/tilt mast and the Hazcams are hard-mounted directly to the rover body. All 9 cameras have been extensively used during the surface operations phase of the mission.

Navigation planning and Rover localization are performed primarily with Navcam panoramas and single frame images, although in the cases where long-distance 


\begin{tabular}{|c|c|}
\hline Cameras & $\begin{array}{l}\text { MER Engineering Camera Description } \\
\text { and Objectives }\end{array}$ \\
\hline $\begin{array}{l}\text { Descent } \\
\text { Camera }\end{array}$ & $\begin{array}{l}\text { Acquire } 3 \text { images of the Martian surface } \\
\text { between altitudes of } 2000 \mathrm{~m} \text { and } 1200 \\
\text { meters during descent. Field of view: } 45 \\
\text { degrees, 3-meter/pixel spatial resolution. } \\
\text { Broadband, visible filter. }\end{array}$ \\
\hline Navcams & $\begin{array}{l}\text { Provide terrain context for traverse } \\
\text { planning and Pancam, Mini-TES } \\
\text { pointing. } 360 \text {-degree field of regard at }<1 \\
\text { mrad/pixel angular resolution. Stereo } \\
\text { ranging out to } 100 \text { meters }(30 \mathrm{~cm} \text { stereo } \\
\text { baseline). Broadband, visible filter. }\end{array}$ \\
\hline Hazcams & $\begin{array}{l}\text { Provide image data for the onboard } \\
\text { detection of navigation hazards during a } \\
\text { traverse. Provide terrain context } \\
\text { immediately forward and aft of the rover } \\
\text { (in particular the area not viewable by the } \\
\text { Navcams) for traverse planning. Support } \\
\text { Instrument Deployment Device (IDD) } \\
\text { operations. Support Rover fine } \\
\text { positioning near IDD targets. Wide field } \\
\text { of view (120 degrees), } 2 \mathrm{mrad} / \text { pixel } \\
\text { angular resolution. Stereo ranging } \\
\text { immediately in front of the rover (10 cm } \\
\text { stereo baseline) to an accuracy of }+/-5 \\
\text { mm. Broadband, visible filter. }\end{array}$ \\
\hline
\end{tabular}

Table 1. A description of the MER Engineering Cameras, from [2].

\begin{tabular}{|c|c|}
\hline Cameras & $\begin{array}{l}\text { MER Science Camera Description and } \\
\text { Objectives }\end{array}$ \\
\hline Pancam [4] & $\begin{array}{l}\text { Obtain monoscopic and stereoscopic } \\
\text { image mosaics. Obtain multispectral } \\
\text { visible to short-wave near-IR images } \\
\text { over a range of viewing geometries. } \\
\text { Obtain images of the Sun and Martian } \\
\text { sky. Serve as the primary Sun-finding } \\
\text { camera for rover navigation. Resolve } \\
\text { objects on the scale of the rover wheels } \\
\text { to distances of } 100 \mathrm{~m} \text {. Support } \\
\text { acquisition and release of exciting E/PO } \\
\text { products. }\end{array}$ \\
\hline $\begin{array}{l}\text { Microscopi } \\
\text { c Imager [6] }\end{array}$ & $\begin{array}{l}\text { Image fine-scale morphology of natural } \\
\text { surfaces. Image fine-scale texture and } \\
\text { reflectance of abraded surfaces. Aid in } \\
\text { the interpretation of data gathered by } \\
\text { other instruments by imaging areas } \\
\text { examined by them at high resolution. }\end{array}$ \\
\hline
\end{tabular}

Table 2. A description of the MER Science Cameras (Pancam [4] and Microscopic Imager [6]). traverse designation is desired, the higher-resolution Pancam cameras [4] are used. Operation of the rover's robotic arm, also called the Instrument Deployment Device (IDD) [5], is done with Front Hazcam imagery. Autonavigation is performed with either Navcam or Hazcam imagery. In addition to the engineering functions described above, all of the cameras are used for purposes of scientific investigation, with the Pancam [4] and Microscopic Imager [6] acquiring the majority of scientific image data.

Because of their operational uses, the cameras are grouped into two types: "engineering" cameras and "science" cameras. All of the MER cameras share the same electronics design [2] and differ only in their optical properties (with the exception of the descent imager, which has a slightly different video gain). Tables 1 and 2 show the operational descriptions of the engineering and science cameras, respectively.

\subsection{Hardware}

All of the MER Cameras were built at the Jet Propulsion Laboratory (JPL) in Pasadena, CA, under a fast-paced 32month development schedule. The camera designs were developed as a collaborative effort between members of the MER team at JPL and MER science team members at Cornell University in Ithaca, NY and the United States Geological Survey (USGS) in Flagstaff, AZ. For details about the camera hardware, see [2].

\subsection{Flight Software}

The MER onboard flight imaging software is described in detail in [7]. The onboard image processing capabilities of the flight system are extensive and include: autoexposure, image subframe extraction, image spatial downsampling, and image compression. The system also includes the capability to point the mast-mounted cameras in a number of surface-based coordinate frames and at selected astronomical targets such as the sun and the Martian moons.

For every image acquired by the rover, the MER Imaging Services flight software generates a small (64 x 64 pixel) "thumbnail image". These thumbnail images are sent back to Earth at a relatively high priority and are usually downlinked the same day. The thumbnail images allow ground operators to preview an image before the full image is downlinked. This opens the possibility for the operators to verify the success of certain imaging acquisitions and confirm to the rest of the operations team that the rover can move to the next target before the full images are received (receipt of the full images often happens several days later). The thumbnails are also sometimes used to reprioritize image data for higher (or lower) downlink transmission.

\subsection{Ground Software}

Aspects of the MER ground image processing software are described in detail in [2], [8] and [9]. The ground image processing software decompresses the image data, assembles ancillary information into the image header, and writes the image to an Experiment Data Record (EDR) file 
in PDS (Planetary Data System) format. The raw EDR files are processed further into Reduced Data Record (RDR) files. The RDR file types are numerous and include stereo correlation maps, mosaics, and 3-dimensional terrain meshes.

\section{MER Surface Imaging Operations}

The MER cameras have returned over 73,000 images as of 13 March 2005 (table 3). The camera hardware (and associated software) has functioned nominally since landing. The cameras and flight software have been utilized as expected, and the ground software has performed extremely well - over 750,000 derived image products have been generated on the ground [8].

The majority of the images acquired during a typical sol on Mars are scientific in purpose. This paper discusses the images acquired primarily for operational purposes. The operational images comprise approximately $20 \%$ of the total number of images returned by the rovers.

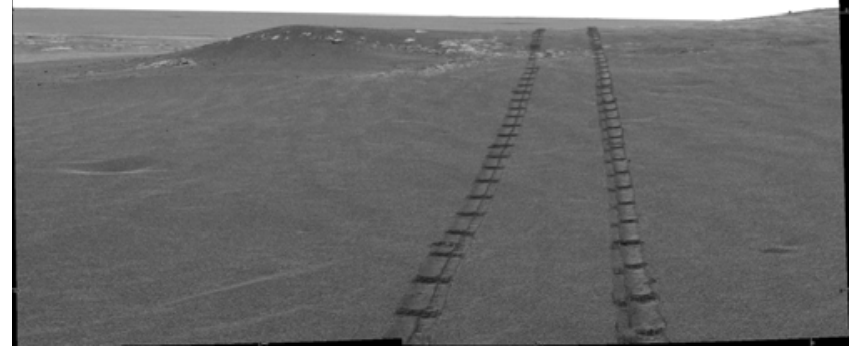

Figure 2. Post-drive Pancam 2x1 mosaic from Opportunity, acquired shortly after Endurance crater egress.

\subsection{Operational Image Totals}

\begin{tabular}{|l|r|r|}
\hline & $\begin{array}{l}\text { Spirit Image } \\
\text { Totals (number } \\
\text { of images) }\end{array}$ & $\begin{array}{l}\text { Opportunity } \\
\text { Image Totals } \\
\text { (number of } \\
\text { images) }\end{array}$ \\
\hline Descent Camera & 3 & 3 \\
\hline Navcam & 5,666 & 5,132 \\
\hline Front Hazcam & 3,089 & 2,036 \\
\hline Rear Hazcam & 26,138 & 25,432 \\
\hline Pancam & 1,947 & 2,578 \\
\hline $\begin{array}{l}\text { Microscopic } \\
\text { Imager }\end{array}$ & 37,639 & 35,653 \\
\hline Total & 89 & 88 \\
\hline $\begin{array}{l}\text { Average number } \\
\text { of images per sol }\end{array}$ & & \\
\hline $\begin{array}{l}\text { Total for both } \\
\text { rovers }\end{array}$ & 73,291 \\
\hline
\end{tabular}

Table 3. Images totals for both MER rovers, as of 13 March 2005 (i.e., Sols 423-A and 403-B)

\subsubsection{Uplink Process and Sol Types}

Camera commands are generated on Earth and sent to the Rovers on Mars as part of the MER 10-hour daily uplink process. Each rover has a dedicated uplink team per sol, and the two teams operate independently of one another on any given sol. The uplink process begins upon examination of the most recent image data from the rovers, typically performed within hours (sometimes minutes) of data receipt on Earth. During the MER primary mission (Sols 1 through 90) the generation of the camera commands (approximately 150 per sol, per rover) required 3 operations personnel per sol, per rover. The introduction of efficiencies and software automation has reduced the number of camera sequencing personnel to 2 operations personnel per day, per rover. We have considered the possibility of utilizing 1 operations personnel per day per rover, but the size and complexity of a typical imaging sol will likely preclude this from happening in the near future.

Based on the particular terrain for any given sol (and the science objectives for that sol), the types of rover activities fall into three main sol types (or combinations thereof):

1) Drive Sol: mobility system activities

2) IDD Sol: movement of robotic arm

3) Remote Sensing Sol: no arm or rover mobility system activities

\subsection{Drive Sols}

For the first 423 sols of the Spirit surface mission, approximately 192 Sols (45\%) were drive sols (table 4). An average of 134 images per sol were acquired on drive sols. The Opportunity totals are similar - for the first 403 Sols of the Opportunity surface mission, approximately 178 Sols (44\%) were drive sols (table 4). An average of 111 images per sol were acquired on Opportunity drive sols.

\begin{tabular}{|l|l|l|}
\hline & Spirit & Opportunity \\
\hline Number of Drive Sols & 192 sols & 178 sols \\
\hline $\begin{array}{l}\text { Percentage of total } \\
\text { sols }\end{array}$ & $45 \%$ & $44 \%$ \\
\hline $\begin{array}{l}\text { Average number of } \\
\text { images per drive sol }\end{array}$ & 134 & 111 \\
\hline
\end{tabular}

Table 4. Number of drive sols for the first Earth year of surface operations, and average number of images acquired per drive sol, for both MER rovers.

The images acquired on a drive sol include a standard set of "end of drive" stereo image data, acquired at the end of a traverse. This set of data typically includes a set of stereo Front and Rear Hazcam images, a stereo Navcam panorama, and an optional set of stereo Pancam images in the future (upcoming sol) drive direction. In addition to the ultimate (final) set of Front/Rear Hazcam images, a penultimate (roughly 1 meter before the final position) set of Front/Rear Hazcam images are often acquired. 


\subsubsection{Penultimate Front Hazcams}

Prior to arriving in the final position at the end of a traverse, a penultimate Front Hazcam stereo pair is often acquired. This image set provides a view of the area that the rover drives into (and parks on) as part of the final drive step (see figure 3). Because the deploy space of the IDD is not fully visible from any of the cameras at the ultimate position, the penultimate set is required to determine if there are any obstructions in the IDD workspace that would prevent the IDD from safely deploying.

Figure 4 shows a Penultimate Front Hazcam from Opportunity, Sol 399. The Penultimate Front Hazcams are

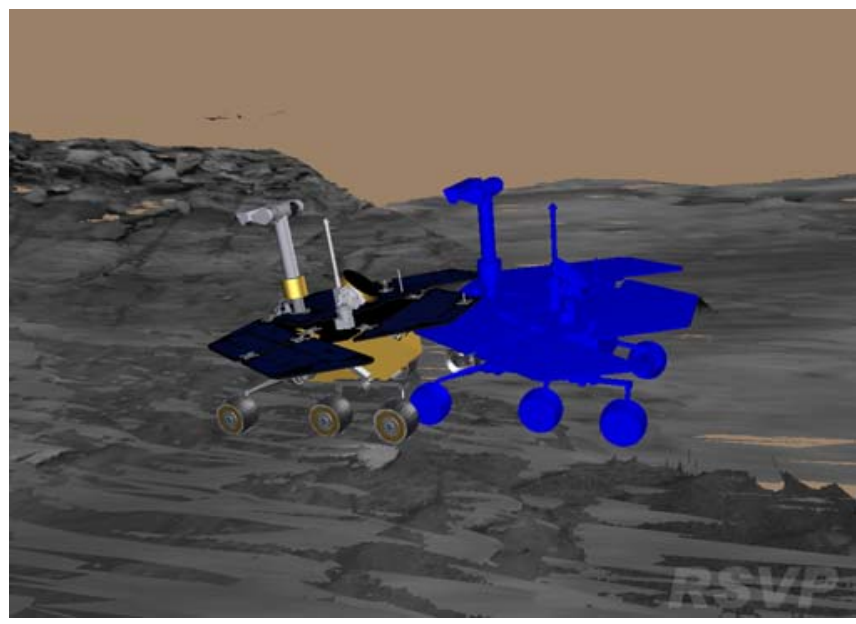

Figure 3. Image showing the penultimate (blue) and ultimate (color) rover positions. This figure was created by the software described in [10].

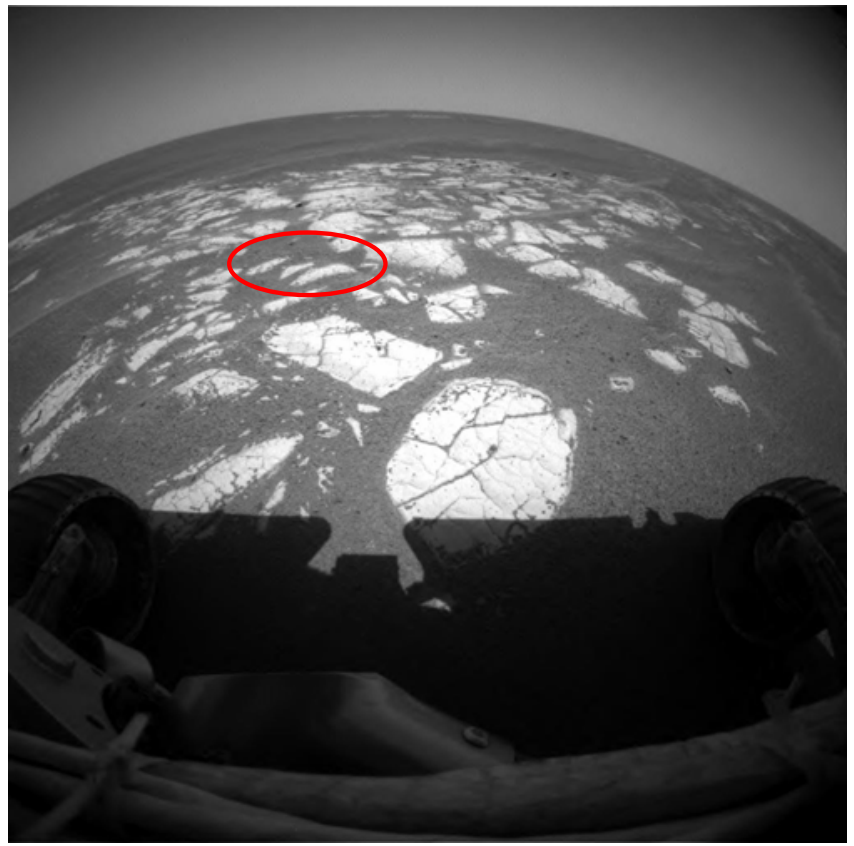

Figure 4. A Penultimate Front Hazcam image from Sol 399-B, compressed at 0.5 bits/pixel more heavily compressed than the typical image compression rates of 0.5 bits/pixel to $1 \mathrm{bit} /$ pixel are typically used for this image data.

\subsubsection{Ultimate Front Hazcams}

Upon arrival at the final rover position for a given sol, an Ultimate (final) Front Hazcam stereo pair is almost always acquired. These images are used for near-field hazard determination as well as IDD target designation on the following sol (if applicable).

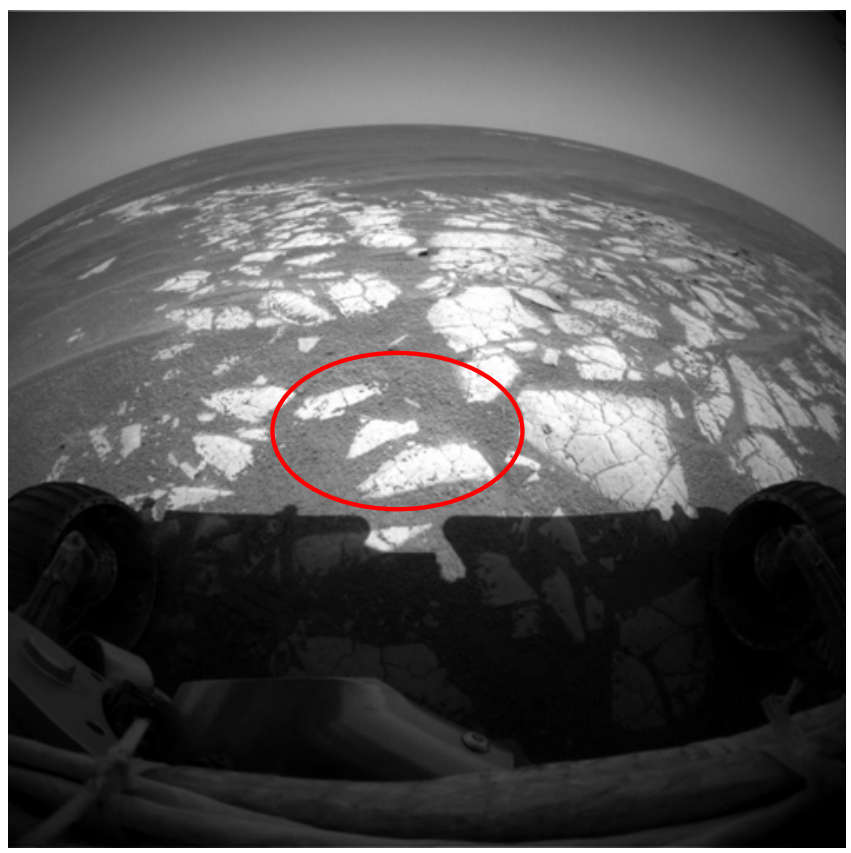

Figure 5. The Ultimate Front Hazcam image from Sol 399$\mathrm{B}$ that corresponds to the Penultimate image shown in figure 4. This image is compressed at a rate of 4 bits/pixel. Note how the area in the middle of the image in figure 4 is now under the rover. The red oval in both images shows the location of the same surface features.

Because IDD operations require accurate (i.e., better than +/- $0.5 \mathrm{~cm}$ RMS error) 3-D terrain knowledge, the Ultimate Front Hazcam images usually undergo light compression the images are typically compressed at a rate of 4 bits/pixel, which is close to lossless compression. Figure 5 shows an Ultimate Front Hazcam image.

\subsubsection{Ultimate and Penultimate Rear Hazcam Images}

Rear Hazcam images acquired at the end of traverses are useful for determining the nature of the terrain around the rear of the rover, particularly the areas not visible from the Navcams. They also often provide excellent views of rover tracks. 


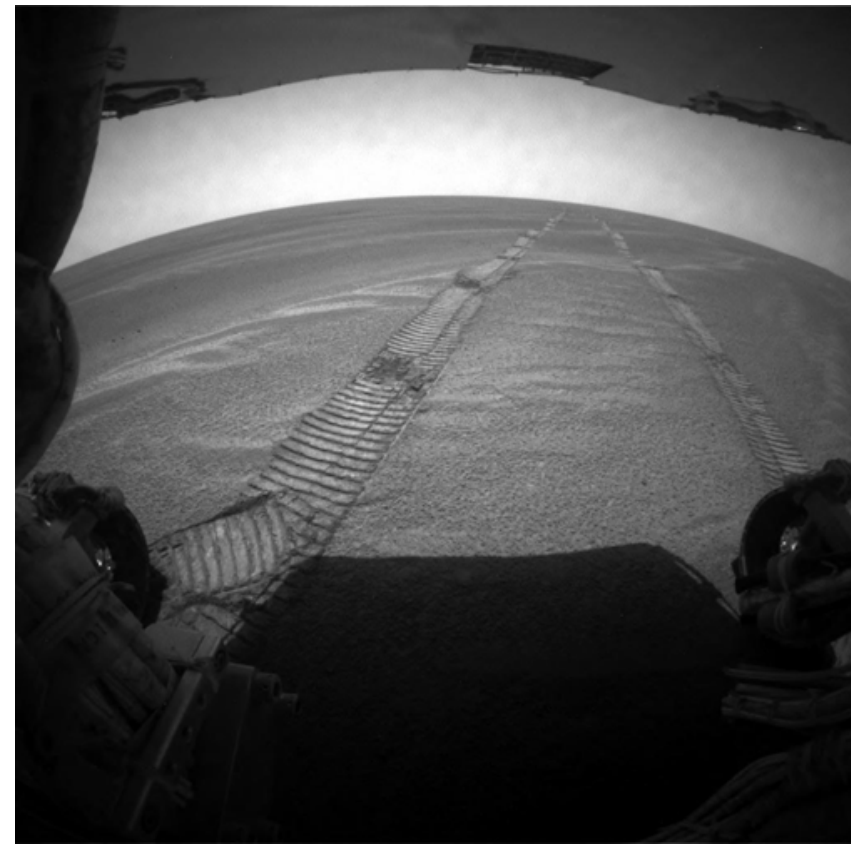

Figure 6. An Ultimate Rear Hazcam image from Sol 399-B compressed at 2 bits/pixel. The track at the lower left indicates that the rover had performed a "turn to comm." maneuver, which optimizes the geometry for UHF transmission to the Mars orbiting communication links. The Rear Hazcam images often offer good views of the rover tracks, and are often examined in detail by soil scientists on the team.

The presence of tracks in the Rear Hazcam images varies from sol to sol and depends on the nature of the traverse (forward vs. backward driving), whether or not the final orientation of the rover has been optimized for UHF transmission (via a "turn to comm." maneuver), and whether or not the downlink volume for the sol allows for the acquisition of these images. Figure 6 shows an Ultimate Rear Hazcam image. Ultimate rear Hazcam images are typically compressed at a rate of $2 \mathrm{bits} /$ pixel. As with the other Hazcam images, the actual compression rate varies from sol to sol, depending on the operational context and nature of the terrain.

\subsubsection{Navcam Drive Direction Imaging}

Navcam images are used extensively for traverse planning, localization, and as targeting maps for the science instruments (i.e., Pancam and Mini-TES). A typical end of drive Navcam image set includes a 360 x 45-degree panorama (i.e., 10 images wide by 1 image high). The "drive direction" portion of the panorama is typically compressed at higher bit rates (i.e., less compression) than the rearward-looking portion of the panorama.

Not all Navcam panoramas are 360-degrees in extent. Constrained downlink volumes will often limit the size of a Navcam panorama. If a traverse is particularly short, the panorama is sometimes only 3 images wide by 1 image high, or in some cases even smaller.

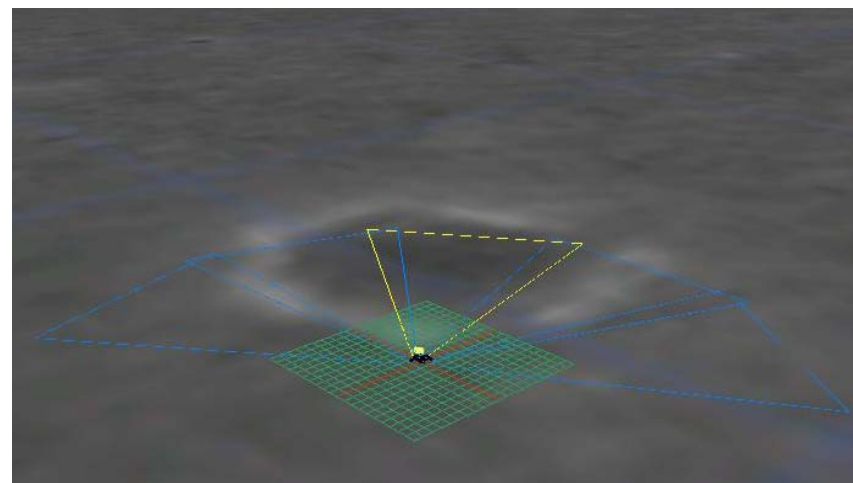

Figure 7. An overhead oblique view of a 5x1 Navcam "drive direction" panorama. The center image (yellow footprint) tends to be the most critical image in the panorama and is acquired with a higher downlink priority than the other four images (shown as blue footprints). This prioritization scheme enables the center image to arrive at Earth ahead of the other images in the panorama. The images in a forward-looking panorama are typically compressed at bit rates of 2-4 bits/pixel, depending on the terrain and operational context. This figure was created using the software described in [10].

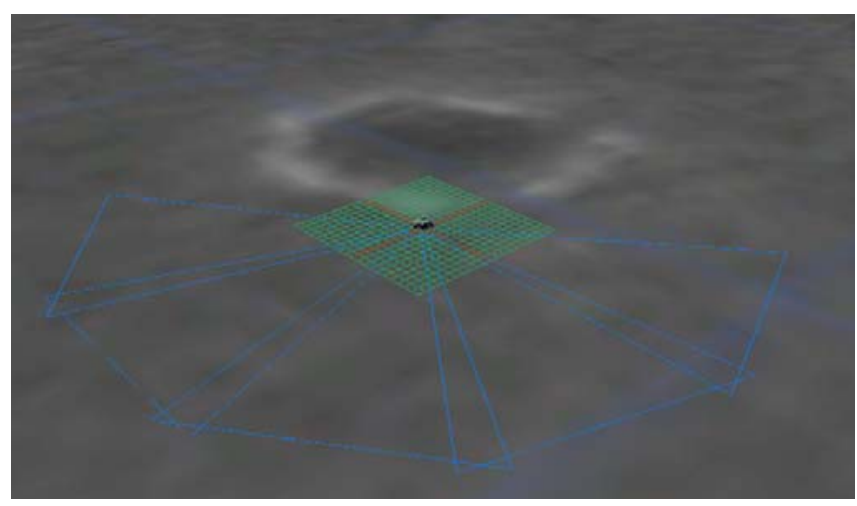

Figure 8. An overhead oblique view of a $5 \times 1$ "rearward looking" panorama. The rearward looking panoramas are generally acquired for purposes of localization. Because they are not as operations critical, the images are generally given a lower downlink priority than the forward-looking portion of the panorama and are typically compressed at rates of $1 \mathrm{bits} / \mathrm{pixel}$ to $2 \mathrm{bits} / \mathrm{pixel}$. This figure was created using the software described in [10].

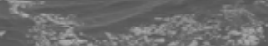

Figure 9. A full 360-degree Navcam panorama, acquired on Sol 399-B, near Vostok crater, shown in a cylindrical projection. This panorama contains both the forward and rearward-looking portions of the panoramas depicted figures 7 and 8 .

The forward and rearward portions of a Navcam panorama are assembled on Earth into a 360-degree 
mosaic. The panorama depicted in figures 7 and 8 is shown as an assembled mosaic in figure 9.

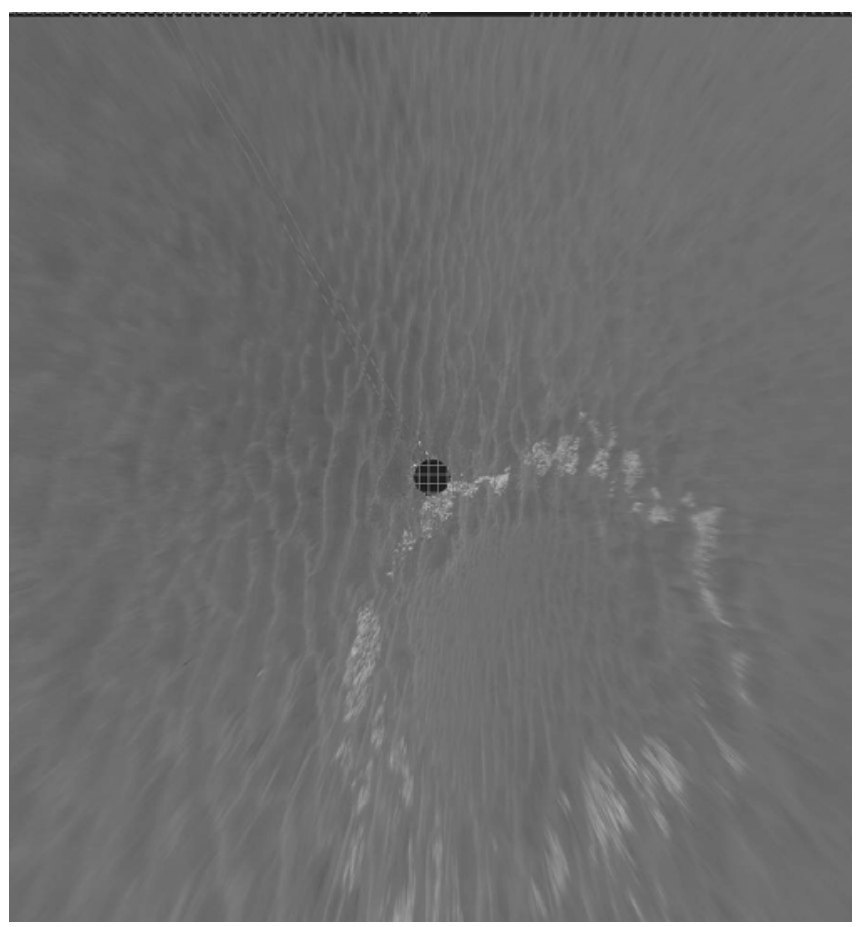

Figure 10. The same Navcam panorama of figure 9, shown as a vertical projection mosaic.

\subsection{IDD Sols}

For the first 423 sols of the Spirit surface mission, approximately 190 Sols (45\%) were of the IDD sol type (table 5). An average of 100 images per sol were acquired on these sols. The Opportunity totals are slightly higher due to extensive IDD activity in Endurance crater - for the first 403 Sols of the Opportunity surface mission, approximately 210 Sols (52\%) were IDD sols. An average of 104 images per sol were acquired on Opportunity IDD sols (see table 5).

\begin{tabular}{|l|l|l|}
\hline & Spirit & Opportunity \\
\hline Number of IDD Sols & 190 sols & 210 sols \\
\hline $\begin{array}{l}\text { Percentage of total } \\
\text { sols }\end{array}$ & $45 \%$ & $52 \%$ \\
\hline $\begin{array}{l}\text { Average number of } \\
\text { images per IDD sol }\end{array}$ & 100 & 104 images/sol \\
\hline
\end{tabular}

Table 5. Number of IDD sols, and average number of images acquired per IDD sol, for both MER rovers.

Operation of the IDD is done primarily through the use of Front Hazcam imagery. The Ultimate Front Hazcam images (see figure 5) are used as the source data for 3-D terrain mesh generation and provide the data necessary for IDD command visualization. The Front Hazcams are also used to document the actual placement of the IDD onto the selected targets. Because these images are predominantly for documentation purposes only, they are generally compressed at a rate of $1 \mathrm{bit} /$ pixel and are often spatially downsampled at half-resolution. Figure 11 shows an example documentation image.

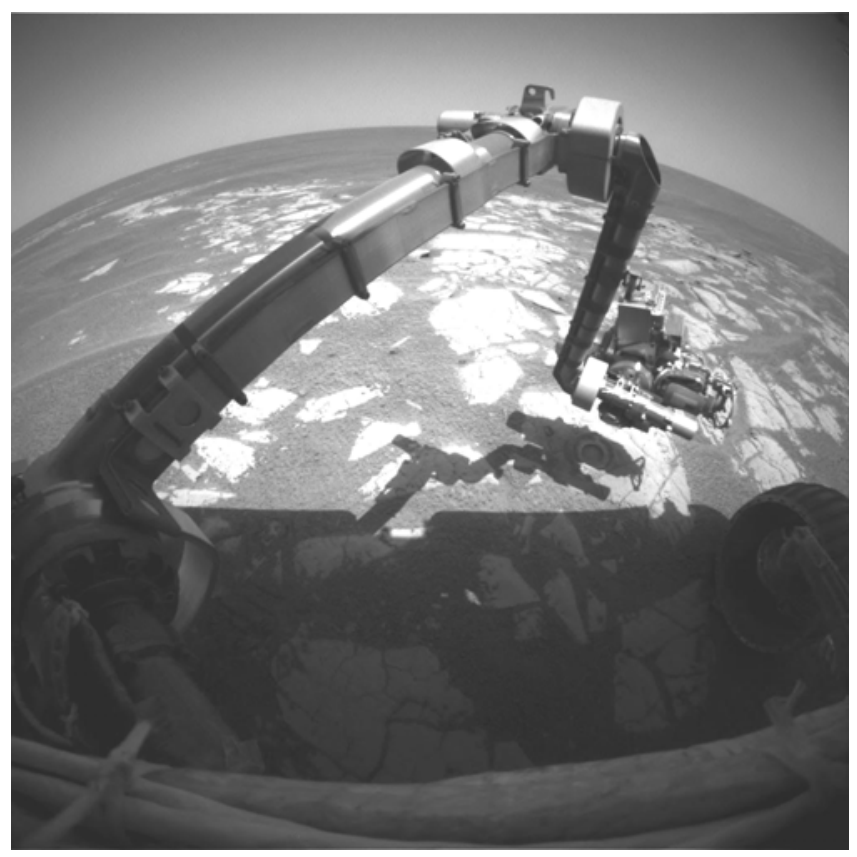

Figure 11. IDD documentation image, showing the placement position of the IDD onto a target. This particular image shows the Microscopic Imager placement above a Rock Abrasion Tool (RAT) hole, acquired on Sol 403-B.

\subsection{Drive/IDD Combination Sols}

A smaller group of Sols involve both driving and IDD activities. This sol type occurred on 70 sols for Spirit and on 76 Sols for Opportunity. The image averages are shown in table 6.

\begin{tabular}{|l|l|l|}
\hline & Spirit & Opportunity \\
\hline $\begin{array}{l}\text { Number of } \\
\text { combination Sols }\end{array}$ & 70 sols & 76 sols \\
\hline $\begin{array}{l}\text { Percentage of total } \\
\text { sols }\end{array}$ & $17 \%$ & $19 \%$ \\
\hline $\begin{array}{l}\text { Average number of } \\
\text { images per IDD sol }\end{array}$ & 139 & 123 images/sol \\
\hline
\end{tabular}

Table 6. Number of Drive/IDD combination sols, and average number of images acquired per combination sol, for both MER rovers.

\section{MER Imaging System Performance}

As of 13 March 2005, the performance of the MER Imaging system on the surface of Mars has been nominal. The Mars Exploration Rover (MER) camera suite continues to perform to within specifications. Image signal to noise ratios (SNRs) are greater than 150:1, camera flat fields remain stable, and single-pixel degradation has been negligible. 


\subsection{Dust Contamination}

On Sol 331, during the Opportunity Heatshield observation campaign, Rear Hazcam (both left and right) images started exhibiting a mottled pattern (i.e., a spotted, billowing pattern) in the sky portion of the image. The previous image (from Sol 330) does not show this effect. Subsequent Rear Hazcam images show that the mottled pattern is virtually identical from Sol to Sol, suggesting that the pattern is an instrument effect and not an atmospheric feature. The pattern does not appear in any of the Front Hazcam, Navcam, Pancam, or MI camera images.

On Sol 417, Spirit's Front Hazcam images also showed a contamination pattern consistent with dust adhesion onto the surface of the lenses. The patterns appeared suddenly on Sol 417 and did not exist on Sol 416 or earlier. The contamination pattern is similar to the patterns that appeared on the Opportunity Rear Hazcams, but the Spirit contamination is more pronounced. Peak to peak DN differences for the Spirit spots (i.e., the DN value of a dark spot subtracted from the neighboring sky background) are around $120 \mathrm{DN}$, vs. around $100 \mathrm{DN}$ for Opportunity. The Spirit spots are also slightly bigger than Opportunity (17-18 pixels in diameter on Spirit vs.15-16 on Opportunity). The Spirit dust contamination appears to be related to an increase in local wind activity.

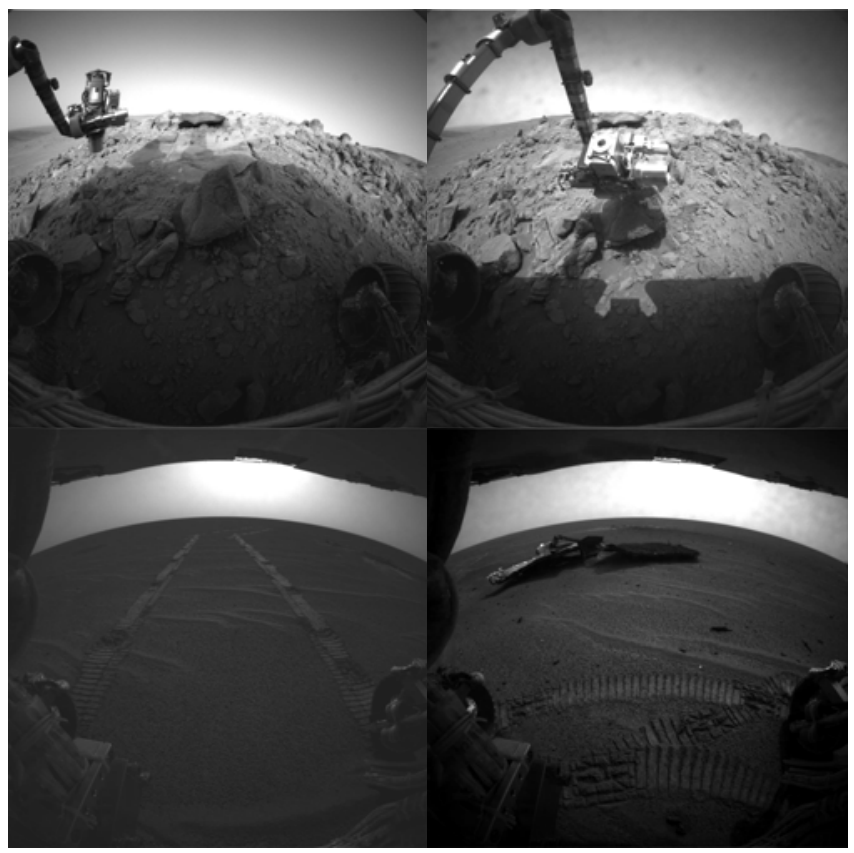

Figure 12. Dust contamination on the Spirit Front Hazcams (top) and the Opportunity Rear Hazcams (bottom). The images on the left show the image quality before dust contamination and the images on the right show the image quality immediately after dust contamination.

\section{Conclusions}

The MER Imaging System was designed to be a flexible and capable component of the MER Rover system. Over
73,000 images later, the original design goal has been achieved.

\section{Acknowledgements}

The work described in this paper was carried out at the Jet Propulsion Laboratory, California Institute of Technology, under a contract with the National Aeronautics and Space Administration.

\section{References}

[1] Crisp, J. A., M. Adler, J. R. Matijevic, S. W. Squyres, R. E. Arvidson, and D. M. Kass, "Mars Exploration Rover mission”, J. Geophys. Res., 108(E12), 8061, 2003.

[2] Maki, J. N., et al., "Mars Exploration Rover Engineering Cameras", J. Geophys. Res., 108(E12), 8071, 2003.

[3] S. W. Squyres, et al., "In Situ Evidence for an Ancient Aqueous Environment at Meridiani Planum, Mars” Science 3 December 2004; 306: 1709-1714.

[4] Bell, J. F., III, et al., Mars Exploration Rover Athena Panoramic Camera (Pancam) investigation, J. Geophys. Res., 108(E12), 8063, 2003.

[5] Squyres, Steven W., et al., Athena Mars rover science investigation, J. Geophys. Res., 108(E12), 8062, 2003.

[6] Herkenhoff, K. E., et al., Athena Microscopic Imager investigation, J. Geophys. Res., 108(E12), 8065, 2003.

[7] Litwin T. E., and Maki, J. N., "Imaging Services Flight Software on the Mars Exploration Rovers", submitted to 2005 IEEE International Conf. on Systems, Man, and Cybernetics, Waikoloa, HI.

[8] D. Alexander, P. Zamani, R. Deen, P. Andres, H. Mortensen, "Automated Generation of Image Products for Mars Exploration Rover Mission Tactical Operations", submitted to 2005 IEEE International Conf. on Systems, Man, and Cybernetics, Waikoloa, HI.

[9] R.G. Deen, J.J. Lorre, "Seeing in Three Dimensions: Correlation and Triangulation of Mars Exploration Rover Imagery", submitted to 2005 IEEE International Conf. on Systems, Man, and Cybernetics, Waikoloa, HI.

[10] F. Hartman et al., "Data Visualization for Effective Rover Sequencing," submitted to 2005 IEEE International Conf. on Systems, Man, and Cybernetics, Waikoloa, HI. 